\title{
High-Level Scientific Programming with Python
}

\author{
Konrad Hinsen ${ }^{1}$ \\ Centre de Biophysique Moléculaire (UPR 4301 CNRS), Rue Charles Sadron, 45071 \\ Orléans Cedex 2, France \\ hinsen@cnrs-orleans.fr \\ WWW home page: http://dirac.cnrs-orleans.fr/
}

\begin{abstract}
Scientific computing is usually associated with compiled languages for maximum efficiency. However, in a typical application program, only a small part of the code is time-critical and requires the efficiency of a compiled language. It is often advantageous to use interpreted high-level languages for the remaining tasks, adopting a mixed-language approach. This will be demonstrated for Python, an interpreted objectoriented high-level language that is particularly well suited for scientific computing. Special emphasis will be put on the use of Python in parallel programming using the BSP model.
\end{abstract}

\section{Introduction}

Scientific computing has some specific requirements that influence the choice of programming tools. The most outstanding property of scientific computing is its explorative nature: although some standard methods are used over and over again, they are used in different combinations every time, and often it is necessary to add custom algorithms and programs to a collection of well-established standard code. Although the literature on scientific computing leaves the impression that all that matters are efficient number crunching and visualization methods, the day-to-day work of a computational scientist involves a lot of interfacing, file format conversion, bookkeeping, and similar tasks, often made difficult by bad user interface design and lack of documentation. These lengthy and unattractive tasks often discourage scientists to pursue a potentially interesting idea. Good programming tools can thus make an important contribution to good computational science.

High-level languages can help in several ways. At the simplest level, they can be used to write all tools that are not time critical, such as simple analysis programs, file format converters, etc. As a general rule, high-level languages are much better suited for I/O- and text-oriented tasks than the standard programming languages used in scientific computing (Fortran, C, C++). However, as this article will show, they can be useful in number-crunching applications as well, making them easier to develop and to use. The key to these applications is mixed-language programming, i.e. combining a high-level and a low-level language in order to get the best of both worlds.

P.M.A. Sloot et al. (Eds.): ICCS 2002, LNCS 2331, pp. 691-700, 2002.

(c) Springer-Verlag Berlin Heidelberg 2002 
To avoid misunderstandings, an explanation of the term "high-level" is in order. Most of all, it implies no judgement of quality. High-level languages are by definition those whose constructs and data types are close to natural-language specifications of algorithms, as opposed to low-level languages, whose constructs and data types reflect the hardware level. With high-level languages, the emphasis is on development convenience, whereas low-level languages are designed to facilitate the generation of efficient code by a compiler. Characteristic features of high-level languages are interactivity, dynamic data structures, automatic memory management, clear error messages, convenient file handling, libraries for common data management tasks, support for the rapid development of graphical user interfaces, etc. These features reduce the development and testing time significantly, but also incur a larger runtime overhead leading to longer execution times.

Note that what is called "high-level" in this article is often referred to as "very high level"; different authors use different scales. Some authors refer to these languages as "scripting languages", which however seems too limiting, as scripting is merely one of their applications.

The high-level language that is used as an example in this article is Python [1], a language that is becoming increasingly popular in the scientific community. Although other suitable languages exist and the choice always involves personal preferences, Python has some unique features that make it particularly attractive: a clean syntax, a simple but powerful object model, a flexible interface to compiled languages, automatic interface generators for $\mathrm{C} / \mathrm{C}++$ and Fortran, and a large library of reusable code, both general and scientific. Of particular importance is Numerical Python [5], a library that implements fast array operations and associated numerical operations. Many numerical algorithms can be expressed in terms of array operations and implemented very efficiently using Numerical Python. Moreover, Numerical Python arrays are used at the interface between Python and low-level languages, because their internal data layout is exactly that of a $\mathrm{C}$ array.

\section{Scripting and Computational Steering}

A typical situation in computational science is the following: an existing program contains all the revelant methods, but its user interface is cumbersome, I/O facilities not sufficient, and interfacing with other programs could be easier. Another common case is the existence of a library of computational algorithms which is used by relatively simple application programs that are constantly modified. In this case, modification and testing of the applications often take a significant amount of time.

A good solution in both situations is the use of a high-level language for scripting, which is sometimes called computational steering in the context of scientific computing. The user of a Python-scripted application/library writes simple Python programs that make heave use of calls to the application/library, but can also use other Python modules, e.g. for I/O. The advantage of script- 
ing is increased flexibility, e.g. being able to use variables and loops to define calculation paramters, and shorter development and testing times for specific application programs. It is also rather easy to add a graphical user interface.

In case of an existing monolithic program, the first step would be to isolate the computational parts of the code and turn them into a (highly specialized) library; much of the user interface and I/O code would be discarded. This library then has to be provided with a Python interface; in most cases this task can be handled by an automatic interface generator such as SWIG [2] for $\mathrm{C} / \mathrm{C}++$ and Pyfort [3] or F2PY [4] for Fortran.

Another possibility, preferred when scripting is not the standard user interface, is embedding Python into an existing application. This option is limited to $\mathrm{C}$ and $\mathrm{C}++$ programs. A program with an embedded Python interpreter can ask the interpreter to execute code, run a script, etc. A typical case would be a program with a graphical user interface that also offers scripting for advanced users. The difference to the straightforward scripting approach described above is that the application program is in charge of script execution, it can decide if and when to run Python code.

An advantage of the scripting approach is that it is easy to realize. Existing code can be used without extensive modifications, and users need only learn the basics of Python in order to be able to profit from scripting. On the other hand, the benefit is mostly limited to the users, developers work much like before. The majority of the code is still written in a low-level language, and the design of the low-level code, especially its data structures, determine the design of the scripting layer.

\section{High-Level Design}

The complementary approach to scripting is to design an application or library for the high-level language, switching to low-level code only for specific timecritical parts. The roles of the two languages are thus inversed, the low-level code is written specifically to fit into the high-level design. The developer can profit from all of the advantages of high-level languages to reduce development and testing time, and - assuming a good programming style - the code becomes more compact and much more readable. However, this approach makes it less straightforward to integrate existing low-level code, unless it takes the form of a library with a well-designed interface. High-level design also requires a good knowledge of Python and object-oriented techniques.

It must be stressed that the result of this approach is very different from simple scripting, and that the practical advantages are significant. In the course of time, a computational scientist can build up a library of problem-specific code, written by himself or obtained from others, that uses the same scientific concepts and abstractions as natural language: numbers, vectors, functions, operators, atoms, molecules, flow fields, differential equation solvers, graphical representations, etc. In low-level code, with or without scripting, everything would have 
to be expressed in terms of numbers and arrays plus functions working on these data.

To give a simple example, suppose you have a large compressed text file containing one number per line and you want to plot a histogram of that number set. In Python this can be written as follows:

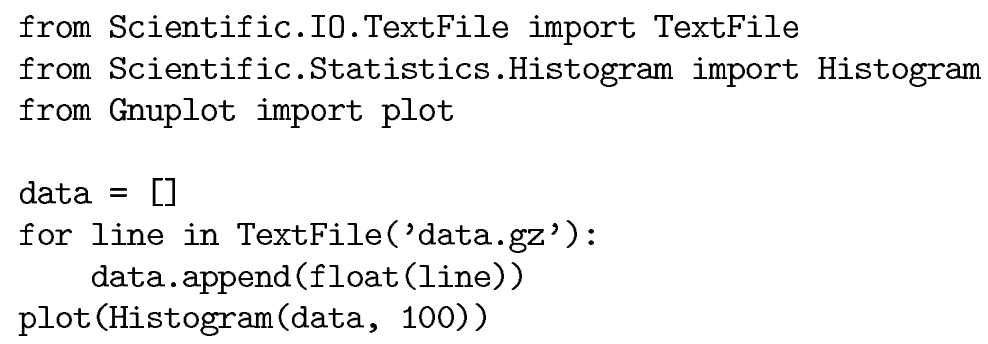

The class TextFile presents a simple abstraction of a text file to the user: a sequence of lines that can be iterated over. Internally it handles many details: it can deal with standard as well as compressed files, and it accepts URLs instead of filenames, in which case it automatically downloads the file from a remote server, stores it temporarily, and deletes the local copy when it has been read completely. The user need not know how any of this is accomplished, for him a text file always remains just a sequence of lines.

The class Histogram provides a similar abstraction for histograms. You give it the data points and the number of bins, and that is all you need to know. Of course the classes TextFile and Histogram must be written first, but only once by one person, they can then be used by anyone anywhere, even interactively, without the need to know how they works internally.

It is often said that object-oriented low-level languages, such as $\mathrm{C}++$, can be used in the same way. However, the higher implementation effort is often discouraging and one settles for the simplest solution that will do the job at hand, even if that means starting from scratch for the next project. Moreover, the code would have to be recompiled for each application, whereas the Python code can be used interactively in a calculator-style fashion. Python with a problem-specific library can be used as a "numerical workbench" for explorative computing.

As a general rule, code reuse works much better in Python than in low-level languages, whose strict typing rules make it difficult to design sufficiently flexible interfaces. With the exception of libraries designed by expert programmers with the explicit goal of generality (e.g. LAPACK), scientific code in low-level languages is almost never directly reusable. In Python, reusability is much easier to achieve, and the weak type compatibility rules, combined with independent name spaces for modules, ensure that even libraries designed completely independently work well together. 


\section{Parallel Computing}

As an example of the use of high-level design in a traditional heavy-duty computing field, this section shows how Python can be used to facilitate the development of parallel programs.

Most textbooks on parallel computing focus on algorithmic aspects, mentioning implementation issues only in passing. However, the implementation of parallel algorithms is far from trivial, since a real-life application uses several different algorithms and requires a significant amount of bookkeeping and I/O. Although many computational scientists envisage parallelization at some time, few ever get beyond simple test programs, because development and debugging become too cumbersome.

A major reason for the difficulty of parallel programming is the low-level nature of the most popular parallel communications library, the Message Passing Interface (MPI). MPI has a large number of functions that permit the optimization of many communication patterns, but it lacks easy-to-use high-level abstractions. Most importantly, it places the responsibility for synchronization fully on the programmer, who spends a lot of time analyzing deadlocks. Moreover, MPI does not offer much support for transferring complex data structures.

A much simpler and more convenient parallel programming model is the Bulk Synchronous Parallel (BSP) model [6]. In this model, computation and communication steps alternate, and each communication step involves a synchronization of all processors, effectively making deadlocks impossible. Another advantage of bundling communication in a special step is the possibility for an underlying communications library to optimize data exchange for a given machine, e.g. by combining messages sent to the same processor. The analysis of algorithms is also facilitated, making it possible to predict the performance of a given algorithm on a given parallel machine based on only three empirical parameters. The Python implementation of BSP (which is part of the Scientific Python package [7]) adds the possibility of exchanging almost arbitrary Python objects between processors, thus providing a true high-level approach to parallelization.

\subsection{Overview}

An important difference for readers familiar with MPI programming is that a Python BSP program should be read as a program for a parallel machine made up of $N$ processors and not as a program for one processor that communicates with $N-1$ others. A Python BSP program has two levels, local (any one processor) and global (all processors), whereas a typical message-passing program uses only the local level. In message-passing programs, communication is specified in terms of local send and receive operations. In a BSP program, communication operations are synchronized and global, i.e. all processors participate.

The two levels are reflected by the existence of two kinds of objects: local and global objects. Local objects are standard Python objects, they exist on a single processor. Global objects exist on the parallel machine as a whole. They have a local value on each processor, which may or may not be the same everywhere. 
For example, a global object "processor id" would have a local value equal to the respective processor number. Global objects also often represent data sets of which each processor stores a part, the local value is then the part of the data that one processor is responsible for.

The same distinction applies to functions. Standard Python functions are local functions: their arguments are local objects, and their return values are local objects as well. Global functions take global objects as arguments and return global objects. A global function is defined by one or more local functions that act on the local values of the global objects. In most cases, the local function is the same on all processors, but it is also common to have a different function on one processor, usually number 0 , e.g. for I/O operations.

Finally, classes can be local or global as well. Standard Python classes are local classes, their instances are local objects, and their methods act like local functions. Global classes define global objects, and their methods act like global functions. A global class is defined in terms of a local class that describes its local values.

Communication operations are defined as methods on global objects. An immediate consequence is that no communication is possible within local functions or methods of local classes, in accordance with the basic principle of the BSP model: local computation and communication occur in alternating phases. It is, however, possible to implement global classes that are not simply global versions of some local class, and that can use communication operations within their methods. They are typically used to implement distributed data structures with non-trivial communication requirements. The design and implementation of such classes requires more care, but they allow the complete encapsulation of both the calculation and the communication steps, making them very easy to use. An example within the Python BSP package is a class that represents distributed netCDF files, and which ensures automatically that each processor handles a roughly equal share of the total data. From a user's point of view, this class has a programming interface almost identical to that of the standard Python netCDF module.

\subsection{Standard Global Classes}

The simplest and most frequent global objects are those which simply mirror the functionality of their local values and add communication operations. They are represented by the classes ParConstant, ParData, and ParSequence, all of which are subclasses of ParValue. The three classes differ in how their local representations are specified.

ParConstant defines a constant, i.e. its local representation is the same on all processors. Example:

$$
\text { zero }=\operatorname{ParConstant}(0)
$$

has a local representation of 0 on all processors.

ParData defines the local representation as a function of the processor number and the total number of processors. Example: 


$$
\text { pid }=\text { ParData (lambda pid, nprocs: pid) }
$$

has an integer (the processor number) as local representation

ParSequence distributes its argument (which must be a Python sequence) over the processors as evenly as possible. Example:

$$
\text { integers }=\text { ParSequence }(\text { range }(10))
$$

divides the ten integers among the processors. With two processors, number 0 receives the local representation $[0,1,2,3,4]$ and number 1 receives $[5,6$, $7,8,9]$. With three processors, number 0 receives $[0,1,2,3]$, number 1 receives $[4,5,6,7]$, and number 2 receives $[8,9]$.

All these classes define the standard arithmetic operations, which are thus automatically parallelized. They also support indexing and attribute extraction transparently.

Global functions are created using the class ParFunction when the local representation is the same local function on all processors (the most common case). Another frequent case is to have a different function on processor 0, e.g. for I/O operations. This is arranged by the class ParRootFunction.

\subsection{A Simple Example}

The first example illustrates how to deal with the simplest common case: some computation has to be repeated on different input values, and all the computations are independent. The input values are thus distributed among the processors, each processor calculates its share, and in the end all the results are communicated to one processor that takes care of output. In the following example, the input values are the first 100 integers, and the computation consists of squaring them.

\section{from Scientific.BSP import ParSequence, ParFunction, I ParRootFunction}

import operator

\# The local computation function.

def square(numbers):

return [ $\mathrm{x} * \mathrm{x}$ for $\mathrm{x}$ in numbers]

\# The global computation function.

global_square $=$ ParFunction(square)

\# The local output function

def output (result):

print result

\# The global output function - active on processor 0 only. global_output $=$ ParRootFunction(output) 


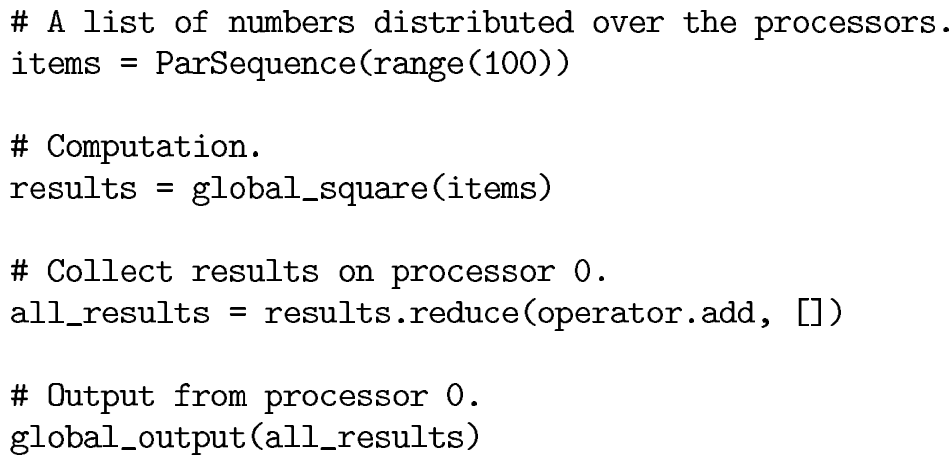

The local computation function is a straightforward Python function: it takes a list of numbers, and returns a list of results. The call to ParFunction then generates a corresponding global function. ParSequence takes care of distributing the input items over the processors, and the call to global_square does all the computation. Before processor 0 can output the results, it has to collect them from all other processors. This is handled by the method reduce, which works much like the Python function of the same name, except that it performs the reduction over all processors instead of over the elements of a sequence. The arguments to reduce are the reduction operation (addition in this case) and the initial value, which is an empty list here because we are adding up lists.

This program works correctly independently of the number of processors it is run with, which can even be higher than the number of input values. However, the program is not necessarily efficient for any number of processors, and the result is not necessarily the same, as the order in which the local result lists are added up by the reduction operation is not specified. If an identical order is required, the processes have to send their processor ID along with the data, and the receiving processor must sort the incoming data according to processor ID before performing the reduction.

One possibly critical aspect of this program is that each processor needs to store all the data initially, before selecting the part that it actually works on. When working with big data objects, it might not be feasible to have each processor store more than the data for one iteration at the same time. This case can be handled with synchronized iterations, in which each processor handles one data item per step and then synchronizes with the others in order to exchange data.

\subsection{Systolic Algorithms}

The next example presents another frequent situation in parallel programming, a systolic algorithm. It is used when some computation has to be done between all possible pairs of data items distributed over the processors. In the example, a list of items (letters) is distributed over the processors, and the computational task 
is to find all pairs of letters (in a real application, a more complex computation would of course be required).

The principle of a systolic algorithm is simple: each data chunk is passed from one processor to the next, until after $N-1$ iterations each processor has seen all data. The new features that are illustrated by this example are general communication and accumulation of data in a loop.

from Scientific.BSP import ParData, ParSequence, ।

ParAccumulator, ParFunction, ParRootFunction, I numberOfProcessors

import operator

\# Local and global computation functions.

def makepairs (sequence1, sequence2):

pairs $=[]$

for item 1 in sequence1:

for item2 in sequence2:

pairs.append ( (item1, item2))

return pairs

global_makepairs $=$ ParFunction(makepairs)

\# Local and global output functions.

def output(result):

print result

global_output $=$ ParRootFunction(output)

\# A list of data items distributed over the processors. my_items = ParSequence ('abcdef')

\# The number of the neighbour to the right (circular). neighbour_pid $=$ ParData (lambda pid, nprocs: [(pid+1)\%nprocs])

\# Loop to construct all pairs. pairs = ParAccumulator (operator. add,[] )

pairs.addValue (global_makepairs (my_items, my_items))

other_items $=$ my_items

for $i$ in range (numberOfProcessors-1):

other_items $=$ other_items.put (neighbour_pid) [0]

pairs.addValue(global_makepairs (my_items, other_items))

\# Collect results on processor 0. all_pairs = pairs . calculateTotal ()

\# Output results from processor 0 . global_output(all_pairs) 
The essential communication step is in the line

other_items $=$ other_items.put (neighbour_pid) [0]

The method put is the most basic communication operation. It takes a list of destination processors (a global object) as its argument; in this example, that list contains exactly one element, the number of the successor. Each processor sends its local representation to all the destination processors.

In the example, each processor receives exactly one data object, which is extracted from the list by a standard indexing operation. The result of the line quoted above thus is the replacement of the local value of other_items by the local value that was stored in the preceding processor. After repeating this $N-1$ times, each processor has seen all the data.

It is instructive to analyze how the systolic loop would be implemented using the popular MPI library in a low-level language. First, either the "items" have to represented by arrays, or appropriate MPI data types need to be defined. Then each processor must send its own data and receive the data from its neighbour. If standard send and receive calls are used, the programmer must take care not to use the same order (send/receive or receive/send) on all processor, as this creates a risk of deadlock. MPI provides a special combined send+receive operation for such cases. The programmer must also allocate a sufficiently large receive buffer, which implies knowing the size of the incoming data. All this bookkeeping overhead would easily exceed the code size of the whole Python program, the programmer would have to take care that it doesn't cause runtime overhead when executing on a single processor, and even more effort would be required to make the program work serially without MPI being present.

\section{Conclusion}

This goal of this article has been to show how a high-level language can be very useful in application domains that are generally considered to be reserved to efficient, compiled low-level languages. The major point to keep in mind is the problem-oriented nature of high-level programming, and its much better possibilities for reusing existing code and for sharing code with other computational scientists.

\section{References}

1. The Python Web site: http://www.python.org/

2. The SWIG Web site: http://www.swig.org/

3. The Pyfort Web site: http://pyfortran.sourceforge.net/

4. The F2PY Web site: http://cens.ioc.ee/projects/f2py2e/

5. The Numerical Python Web site: http://numpy. sourceforge.net/

6. D.B. Skillicorn, J.M.D. Hill and W.F. McColl, "Questions and Answers about BSP", Technical Report PRG-TR-15-96, Oxford University Computing Laboratory, available from http://www.bsp-worldwide.org/implmnts/oxtool.htm

7. The Scientific Python Web site: http://dirac.cnrs-orleans.fr/ScientificPython/ 